\title{
Nondipolar photoionization of atoms
}

\author{
B. Krässig, R. W. Dunford, E. P. Kanter, S. H. Southworth, and L. Young \\ Chemistry Division, Argonne National Laboratory, Argonne, IL 60439, USA
}

\begin{abstract}
Results of the nondipolar angular distribution parameter $\gamma$ are presented for two cases of photoionization with substantially different photon energies, $\mathrm{Kr}$ 1s in the hard $\mathrm{x}$-ray regime, and $\mathrm{He}$ $1 \mathrm{~s}$ in the vuv regime. The nondipolar asymmetries are apparent even at low energies. The presence of dipole and quadrupole resonances strongly modifies the energy dependence of the nondipolar asymmetry parameter $\gamma$. Measurements of $\gamma$ can be used as a spectroscopic tool to characterize quadrupole resonances that are not accessible in photoabsorption measurements.
\end{abstract}

\section{INTRODUCTION}

The vast majority of the phenomena observed in the absorption of photons in matter can be understood on basis of the dipole approximation. In a multipole expansion of the interaction the dipole part is always the dominant one, but at high energies, when the x-ray wavelength approaches the length scale of the atom, the dipole approximation alone may not be sufficient. Yet, the presence of higher multipoles in the photon-atom interaction can be felt even at low photon energies if photoelectron angular distributions are being studied. In particular, the odd-parity dipole interaction and the even-parity quadrupole interaction interfere with each other giving rise to a term in the angular distribution that is odd under inversion. The interference term causes an asymmetry of the angular distribution with respect to the propagation direction of the photon. The interference term vanishes in the integral cross section and it also vanishes in the plane perpendicular to the photon beam where photoelectron angular distributions are most commonly measured. In the following we will describe measurements of the forward-backward asymmetry of the photoelectron angular distribution as a means of accessing information on the nondipolar contents in the interaction. The apparatus used in these measurements exploits special symmetry properties of the photoelectron angular distribution to eliminate the dependence on the polarization characteristics of the photon beam and to reduce instrumental anisotropies. We report results of the nondipolar angular distribution parameter $\gamma$ for $\mathrm{Kr} 1 \mathrm{~s}$ from threshold at $14.3 \mathrm{keV}$ up to $22 \mathrm{keV}$ [1]. As a second example, we present results on the nondipolar asymmetries of $\mathrm{He}$ 1s photoionization with $\sim 60 \mathrm{eV}$ photon energy in the region of the doubly excited autoionization resonances [2]. 


\section{THEORETICAL FORMALISM}

In the quantum mechanical description of the interaction of a photon with an atom the transition operator contains the oscillatory factor $e^{i k \cdot r}$. Here $k$ is the momentum vector of the photon in atomic units and $r$ is the position vector of the ionized atomic electron. In the dipole approximation the exponential is approximated by unity, assuming $k r \ll 1$. Going beyond the dipole approximation, the plane wave can be expanded in partial waves [3] or in multipoles $E 1, M 1, E 2, M 2, \ldots$ [4, 5]. Grouping the expansion terms of orders of $k$ is referred to as the retardation expansion. The leading term in the retardation expansion is unity and thus represents the dipole approximation. It corresponds to the long-wavelength limit of the dipole term, $E 1$, in the multipole expansion. Going to the second term in the retardation series amounts to including the long-wavelength limits of the magnetic dipole, $M 1$, and electric quadrupole, E2. At higher orders the correspondence between terms of the retardation and multipole expansions cannot be made as easily. The multipoles have alternating parities with increasing order $j, \pi(E j)=(-1)^{j}$ and $\pi(M j)=(-1)^{j-1}$, and transfer $j$ units of angular momentum in the interaction. Between electric quadrupole and magnetic dipole the latter is expected to be very weak as it vanishes within the framework of central field calculations [6]. In the present context we will be mainly concerned with electric dipole and electric quadrupole components and the resulting first order retardation correction.

The angular distribution of the emitted photoelectrons depends on the square modulus of the transition matrix element. If the transition operator is written as a coherent sum of two terms, e.g. $T=E 1+E 2$, then the square modulus of the transition matrix element $|\langle T\rangle|^{2}$ will contain cross terms $\langle E 1\rangle\langle E 2\rangle$ in addition to the squares of the pure terms $|\langle E 1\rangle|^{2},|\langle E 2\rangle|^{2}$. The leading retardation correction in the differential cross section beyond the standard dipole approximation contains the $E 1-E 2$ interference term in the short-wavelength limit. This term is of order $k$ and has odd parity which causes a forward-backward asymmetry in the angular distribution. The differential cross section with the first order correction, for linearly polarized photons, can be written as [6]

$$
\frac{d \sigma}{d \Omega}=\frac{\sigma}{4 \pi}\left(1+\beta \mathrm{P}_{2}(\hat{\boldsymbol{\varepsilon}} \cdot \hat{\boldsymbol{p}})+\left(\gamma(\hat{\boldsymbol{\varepsilon}} \cdot \hat{\boldsymbol{p}})^{2}+\delta\right) \hat{\boldsymbol{k}} \cdot \hat{\boldsymbol{p}}\right) .
$$

The quantities $\hat{\boldsymbol{\varepsilon}}, \hat{\boldsymbol{k}}, \hat{\boldsymbol{p}}$ are the unit vectors of the polarization, beam, and electron emission directions, respectively. In addition to the usual anisotropy parameter $\beta$ the differential cross section contains two nondipolar asymmetry parameters, $\gamma$ and $\delta$. In the way these two parameters were defined, $\delta=0$ for the ionization of s electrons.

For ionization of s electrons the asymmetry parameter $\gamma$ depends on the photon energy $\omega$, the radial matrix elements for electric dipole and electric quadrupole transitions $R_{1}, R_{2}$, and the phase differences of the ougoing partial waves from electric dipolar and electric quadrupolar photoionization $\delta_{1}, \delta_{2}[3,6]$

$$
\gamma(n \mathrm{~s})=3 \alpha \omega \frac{R_{2}}{R_{1}} \cos \left(\delta_{2}-\delta_{1}\right)
$$

with $\alpha$ being the fine structure constant.

The second order retardation correction, order $k^{2}$, has even parity and is therefore forward-backward symmetric. The form of the differential cross section including the 


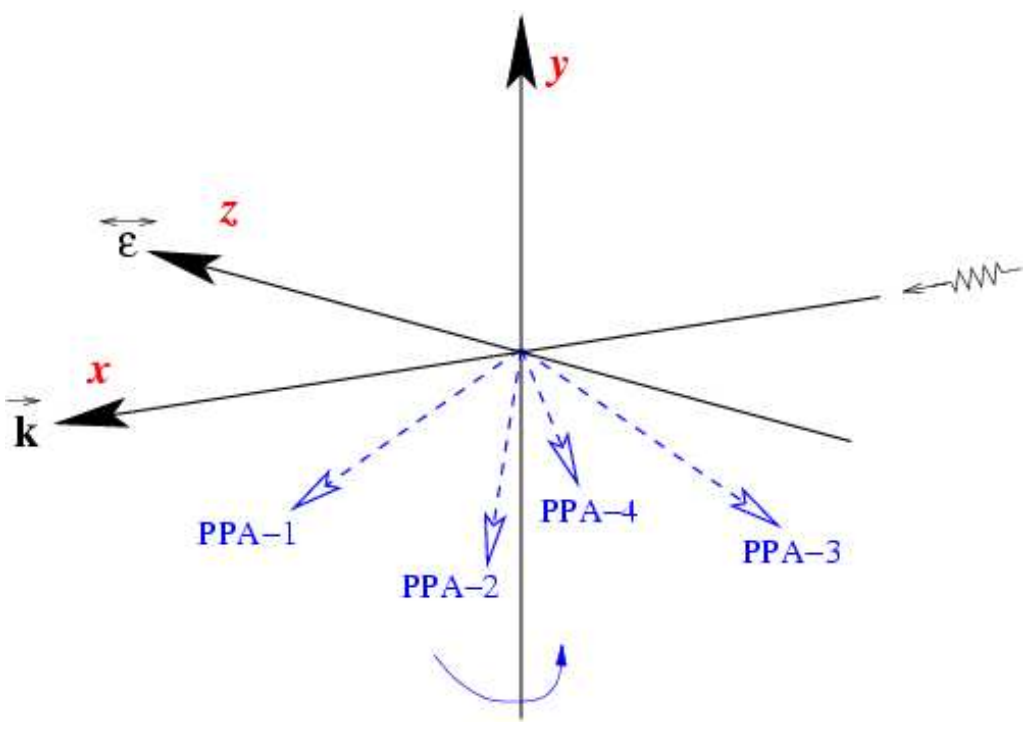

FIGURE 1. Schematic of the positions of the four PPAs relative to the X-ray beam direction $\boldsymbol{k}$ and the polarization direction $\boldsymbol{\varepsilon}$.

second order correction can be found in [7]. The second order correction adds two more independent angular distribution parameters.

\section{EXPERIMENTAL TECHNIQUE}

The apparatus used in the present experiments is a considerably improved version of the one used in our previous experiments [8]. The setup was specifically designed for the measurement of the nondipolar forward-backward asymmetries. The new apparatus utilizes four parallel plate electron analyzers (PPAs) mounted on a common rotation stage. The four PPAs are mounted such that they are aligned along the space diagonals in the cartesian coordinate frame, viewing the interaction region in the center (Fig. 1). In this position the analyzers are at the magic angle $\theta_{m}=54.7^{\circ}$ with respect to all three axes. When the signals of the two analyzers in the forward hemisphere are added together, and the signals of the two backward analyzers are added, then the sum signals are independent of the degree of polarization or the orientation of the polarization vector in the plane perpendicular to the beam axis [9]. The signals are also independent of the anisotropy parameter $\beta$. Furthermore, because the geometry of each analyzer with respect to the interaction region is the same for all four analyzers, the instrumental anisotropies are small. With the rotation stage the individual analyzers can be repositioned so that during a measurement each analyzer collects data at all four positions. In this way the efficiency differences between the analyzers are compensated.

Denoting the signals of the four PPAs at the angular positions shown in Fig. 1 as $I_{1}, I_{2}, I_{3}, I_{4}$, the measured forward-backward asymmetry according to Eq. (1) is propor- 
tional to $\gamma+3 \delta$

$$
\frac{I_{1}+I_{2}-I_{3}-I_{4}}{I_{1}+I_{2}+I_{3}+I_{4}}=\frac{\gamma+3 \delta}{\sqrt{27}}
$$

If the second order retardation correction is taken into account the normalization constant $I_{1}+I_{2}+I_{3}+I_{4}$ in the denominator becomes dependent on one of the second order parameters, $\xi$, [7]

$$
\frac{I_{1}+I_{2}-I_{3}-I_{4}}{I_{1}+I_{2}+I_{3}+I_{4}}=\frac{\gamma+3 \delta}{\sqrt{27}\left(1-\frac{7}{18} \xi\right)} .
$$

The presence of a nonzero $\xi$ therefore affects the result of $\gamma+3 \delta$ if it is extracted from the observed asymmetry using Eq. (3). The values of $\xi$ calculated by Derevianko are generally $\ll 1$ so that the correction in equation (4) is mostly negligible. Also, $\delta=0$ in the two cases of 1 s ionization studied in this report.

\section{RESULTS FOR Kr 1s}

The measurements for the nondipolar asymmetry of 1 s photoionization in krypton were carried out at the BESSRC-CAT 12-ID undulator beam line of the Advanced Photon Source at Argonne National Laboratory. The measurements covered the energy range from $11 \mathrm{eV}$ above the $\mathrm{Kr}-K$ edge to $8000 \mathrm{eV}$ above (x-ray energy $\sim 14.3 \mathrm{keV}-22.3$ $\mathrm{keV})$. A complete account of these measurements will be given elsewhere [1].

Fig. 2 shows the energy dependence of the parameter $\gamma(\mathrm{Kr} 1 \mathrm{~s})$. The data points below $1 \mathrm{keV}$ have negative $\gamma$ indicating preferential backward emission of the photoelectrons. Near $1 \mathrm{keV}$ the nondipolar asymmetry vanishes with $\gamma=0$ and above $1 \mathrm{keV}$ the asymmetry is positive and increasing with energy.

Fig. 2 contains also the prediction of relativistic IPA calculations [10] for this case. The calculation is able to include retardation to all orders. However, in order to demonstrate the degree by which the measurement of $\gamma$ is affected by the omission of the second order contribution we show the result of the first order retardation correction as a full line, and include for comparison the asymmetry resulting from first plus second order retardation corrections. Clearly, the first order calculation reproduces the experimental data very well. The distortion by the presence of the second order correction is less than the experimental error and apparent only at the the highest energies in this measurement.

The energy dependence of the nondipolar asymmetries in $\mathrm{Kr}$ is reminiscent of the ones observed in Ar [8], however with Kr having much larger negative values in the low energy region and the zero crossing of the $\gamma$ parameter being at a much higher energy for $\mathrm{Kr}$. The energy dependences of $\gamma(1 \mathrm{~s})$ for different $Z$ smoothly follow this trend of more negative values and shifting of the zero crossing to higher energies for increasing $Z$. On the low $Z$ end the curves converge on the positive-definite square-root-like energy dependence of the point-Coulombic case of hydrogen. 


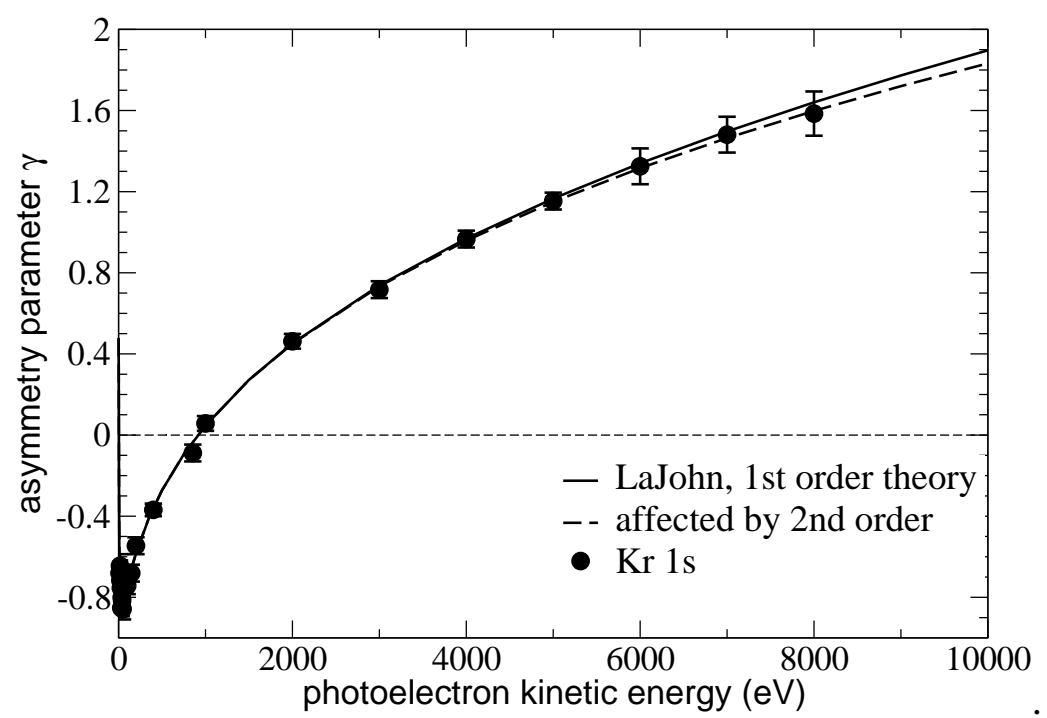

FIGURE 2. Energy dependence of the nondipolar parameter $\gamma$ for $\mathrm{Kr} 1 \mathrm{~s}$ as obtained from Eq. (3). Experimental data, solid circles; relativistic IPA calculation up to 1st order retardation correction [10], full line; calculation up to second order, broken line. See text

\section{RESULTS FOR He 1s}

In helium already the lowest doubly-excited state lies energetically above the ionization energy. The resonantly excited neutral states decay rapidly via autoionization. The interference of the sequential process of excitation followed by decay with the direct process leads to the well known Fano-resonance structure in the energy range between $60.1 \mathrm{eV}$ and the double ionization limit at $79.0 \mathrm{eV}$. The dipole-allowed ${ }^{1} P^{\mathrm{o}}$ series have been studied and characterized with great precision and detail in photoabsorption experiments [11]. The resonance series with couplings and parity other than ${ }^{1} P^{\mathrm{o}}$ are observed in electron scattering experiments [12].

The intent of this measurement was to measure the nondipolar asymmetry at low photon energies and to study the influence of the dipole and (dipole-forbidden) quadrupole autoionization resonances on the nondipolar asymmetry (cf. [13, 14]). The low-energy measurement for helium was performed at the PGM Undulator beam line at the Synchrotron Radiation Center (SRC) of the University of Wisconsin. The complete results of this measurement and a more detailed description can be found in [2].

Fig. 3 shows in the lower panel the signal of an ion detector when scanning the monochromator across the region of the lowest-lying ${ }^{1} D^{\mathrm{e}}$ and ${ }^{1} P^{\mathrm{o}}$ resonances. Only the dipole resonance is observed in the ion signal. A fit to these data, with convolution to the bandpass function of the monochromator, results in values for the dipole resonance parameters that are in excellent agreement with those from the state-of-the-art measurements of this resonance series [11].

In the upper panel of Fig. 3 the nondipolar asymmetry parameter $\gamma$ is shown for the same region. Outside the resonances $\gamma$ has a value of about 0.08 , but $\gamma$ displays pronounced variations at both the dipole and quadrupole resonance energies. In the 

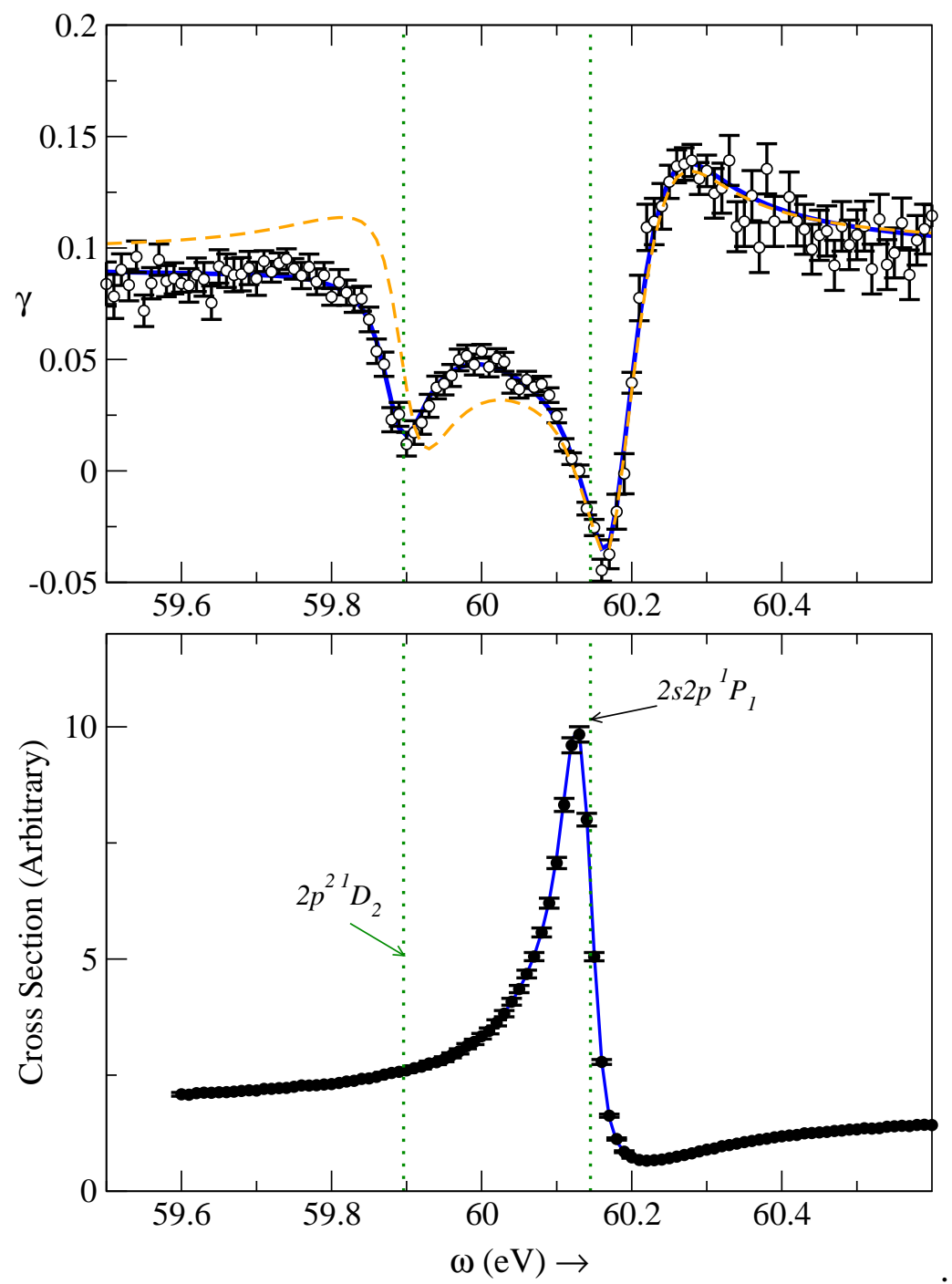

FIGURE 3. Upper panel: Energy dependence of the nondipolar parameter $\gamma$ for $\mathrm{He} 1 \mathrm{~s}$ in the region of the lowest-lying quadrupole and dipole resonances. Experimental data, solid circles; fit to data according to Eq. (5), solid line; curve from Eq. (5) using theoretical values of $q_{2}, \delta_{2}-\delta_{1}$, broken line. Lower panel: energy dependence of the ion signal recorded when scanning across the same energy region. See text

maximum of the dipole resonance $\gamma$ is close to zero because of the predominance of dipole interaction at the maximum. In the minimum of the dipole resonance $\gamma$ has a transient from negative values to positive values. The minimum would be exactly zero for a $\delta$-function-like bandwidth of the monochromator and this, following from Eq. (2), would result in a divergence of $\gamma$ at exactly this energy. With the experimental bandwidth of the experiment this divergence is attenuated to the observed transient.

The functional form of the nondipolar asymmetry in the region of the lowest series of autoionization resonances (one continuum) has been derived by Martin [15]. Starting from Eq. (2) and using the well-established formalism of Fano [16] for both dipole and 
quadrupole,

$$
\gamma^{(r)}=\gamma^{(0)}\left\{\frac{\cos \left(\delta_{2}+\Delta_{2}-\delta_{1}-\Delta_{1}\right)}{\cos \left(\delta_{2}-\delta_{1}\right)}\right\} \times\left\{\frac{q_{2}+\varepsilon_{2}}{\sqrt{1+\varepsilon_{2}^{2}}}\right\} \times\left\{\frac{\sqrt{1+\varepsilon_{1}^{2}}}{q_{1}+\varepsilon_{1}}\right\}
$$

where $\varepsilon_{\ell}=\frac{\omega-\omega_{\ell}}{\Gamma_{\ell} / 2}$ and $\cot \Delta_{\ell}=-\varepsilon_{\ell}$. The superscript $(r)$ on $\gamma$ stands for resonant and $\gamma^{(0)}$ is the nondipolar asymmetry in the absence of the resonances. The quantities with indices $\ell=1,2$ refer to the electric dipole and electric quadrupole interactions, respectively, and concern the Fano $q_{\ell}$ parameter, the resonance widths $\Gamma_{\ell}$, and resonance energies $\omega_{\ell}$. Using Eq. (5) as a fit function to the observed nondipolar asymmetries, further requires proper accounting for the finite bandwith of the monochromator.

By fitting the observed nondipolar asymmetry we obtained the Fano resonance parameters for both the dipole and quadrupole resonances, the off-resonance asymmetry $\gamma^{(0)}$ and also the off-resonant phase shift difference $\cos \left(\delta_{2}-\delta_{1}\right)$ [2]. It is the presence of the resonances with their well-defined resonance phase shifts that allows the separation of $\gamma^{(0)}$ into the radial matrix-element ratio and the phase shift difference, which otherwise would not be possible.

This measurement is the first experimental determination of the Fano resonance parameter $q_{2}\left(2 \mathrm{p}^{21} D^{\mathrm{e}}\right)=-0.25(7)$. This value differs significantly from the theoretical predictions $q_{2}=-1[17,18]$. This quantity is not accessible in scattering experiments. Also the resonance position and width of the quadrupole resonance were determined in this experiment to higher precision than before [2]. The measurement of the nondipolar asymmetry establishes a new spectroscopic tool to characterize accurately the dipoleforbidden quadrupole resonances.

\section{CONCLUSION}

Nondipolar effects are noticeably present in the angular distribution of photoelectrons. The influence of higher electric quadrupole components in the photon beam manifests itself as a forward-backward asymmetry in the angular distribution with respect to the beam direction, leaving the plane perpendicular to the photon beam and angle-integrated cross sections unaffected. In general the nondipolar asymmetries increase with energy, but the individual energy dependences can be strongly influenced by variation of the dipole or quadrupole amplitudes and the phase difference between outgoing partial waves for dipole and quadrupole interactions. As an example we presented data for the energy dependence of $\mathrm{Kr} 1 \mathrm{~s}$ photoelectron up to $8 \mathrm{keV}$ above threshold. The variations of the observed nondipolar asymmetry become very pronounced in the region of resonances. The variation of the nondipolar asymmetry can be used as a spectroscopic tool to characterize quadrupole resonances that are too weak in intensity to be observed directly in photoabsorption. This was shown in the case of helium. Even at photon energies as low as $60 \mathrm{eV}$ the nondipolar asymmetries and the influence of autoionization resonances are distinctly observable. We observed similarly strong variations in the region of the 
cross section minimum in $\mathrm{Xe} 5$ s photoionization near $20 \mathrm{eV}$ photon energy, which will be the subject of a separate publication.

\section{ACKNOWLEDGEMENTS}

We are grateful to N. L. S. Martin, R. Guillemin, O. Hemmers, and D. W. Lindle for their assistance during the experiments at SRC. We thank the BESSRC and SRC staff for excellent research facilities. The University of Wisconsin SRC is funded by the National Science Foundation Grant No. DMR-0084402. This work is supported by the Chemical Sciences, Geosciences, and Biosciences Division of the Office of Basic Energy Sciences, Office of Science, U.S. Department of Energy under contract W-31-109-ENG-38.

\section{REFERENCES}

1. Krässig, B., Bilheux, J., Dunford, R. W., Gemmell, D. S., Hasegawa, S., Kanter, E. P., Southworth, S. H., Young, L., LaJohn, L. A., and Pratt, R. H., Phys. Rev. A (2002), to be published.

2. Krässig, B., Kanter, E. P., Southworth, S. H., Guillemin, R., Hemmers, O., Lindle, D. W., Wehlitz, R., and Martin, N. L. S., Phys. Rev. Lett., 88, 203002 (2002).

3. Bechler, A., and Pratt, R. H., Phys. Rev. A, 39, 1774 (1989).

4. Peshkin, M., "Angular distributions of photoelectrons: Consequences of symmetry", in Advances in Chemical Physics, Vol. XVIII, edited by I. Prigogine and S. A. Rice, John Wiley \& Sons, Inc., 1970.

5. Scofield, J. H., Phys. Rev. A, 40, 3054 (1989).

6. Cooper, J. W., Phys. Rev. A, 47, 1841 (1993).

7. Derevianko, A., Johnson, W. R., and Cheng, K. T., At. Data Nucl. Data Tables, 73, 153 (1999), the parameter $\xi$ corresponds to $\mathrm{V}$ used in this reference, see [19].

8. Jung, M., Krässig, B., Gemmell, D. S., Kanter, E. P., LeBrun, T., Southworth, S. H., and Young, L., Phys. Rev. A, 54, 2127 (1996).

9. Peshkin, M., "Photon beam polarization and nondipolar angular distributions", in Atomic Physics with Hard X-Rays from High Brilliance Synchrotron Light Sources, ANL/APS/TM-16, Argonne National Laboratory, 1996, p. 207.

10. LaJohn, L. A., and Pratt, R. H. (2002), private communication.

11. Schulz, K., Kaindl, G., Domke, M., Bozek, J. D., Heiman, P. A., Schlachter, A. S., and Rost, J. M., Phys. Rev. Lett., 77, 3086 (1996), and references therein.

12. van den Brink, J., Nienhuis, G., van Eck, J., and Heideman, H., J. Phys. B, 22, 3501 (1989), and references therein.

13. Amusia, M. Y., Dolamatov, V. K., and Ivanov, V. K., Sov. Phys. Tech. Phys., 31, 4 (1986).

14. Martin, N. L. S., Thompson, D. B., Baumann, R. P., Caldwell, C. D., Krause, M. O., Frigo, S. P., and Wilson, M., Phys. Rev. Lett., 81, 1199 (1999).

15. Martin, N. L. S. (2001), private communication.

16. Fano, U., Phys. Rev., 124, 1866 (1961).

17. Kheifets, A. S., J. Phys. B, 26, 2053 (1993).

18. Lhagva, O., and Henmedeh, L., J. Phys. B, 27, 4623 (1994).

19. Derevianko, A., Hemmers, O., Oblad, S., Glans, P., Wang, H., Whitfield, S. B., Wehlitz, R., Sellin, I. A., Johnson, W. R., and Lindle, D. W., Phys. Rev. Lett., 84, 2116 (2000). 